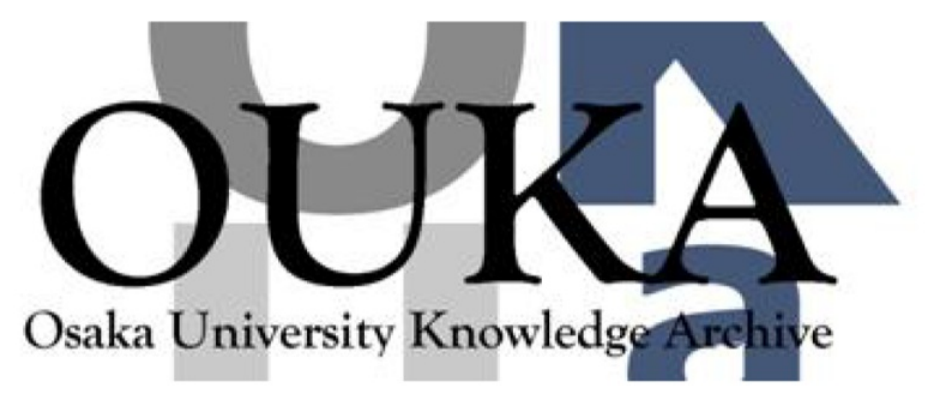

\begin{tabular}{|c|l|}
\hline Title & $\begin{array}{l}\text { GEKK0/HIPER-driven shock waves and equation-of- } \\
\text { state measurements at ultrahigh pressures }\end{array}$ \\
\hline Author(s) & Ozaki, N.; Tanaka, K. A.; Ono, T. et al. \\
\hline Citation & Physics of Plasmas. 11(4) p. 1600-p. 1608 \\
\hline Issue Date & $2004-04$ \\
\hline oaire:version VoR \\
\hline URL & https://hdl. handle. net/11094/3088 \\
\hline rights & \\
\hline Note & \\
\hline
\end{tabular}

Osaka University Knowledge Archive : OUKA

https://ir. Library. osaka-u. ac. jp/

Osaka University 


\title{
GEKKO/HIPER-driven shock waves and equation-of-state measurements at ultrahigh pressures
}

\author{
N. Ozaki, ${ }^{\text {a) }}$ K. A. Tanaka, ${ }^{\text {b) }}$ T. Ono, ${ }^{\text {b) }}$ K. Shigemori, M. Nakai, H. Azechi, and T. Yamanaka \\ Institute of Laser Engineering, Osaka University, Suita, Osaka 565-0871, Japan \\ K. Wakabayashi and M. Yoshida \\ National Institute of Advanced Industrial Science and Technology (AIST), Tsukuba, Ibaraki 305-8565, Japan \\ H. Nagao and K. Kondo \\ Materials and Structures Laboratory (MSL), Tokyo Institute of Technology, Yokohama, \\ Kanagawa 226-8503, Japan
}

(Received 4 September 2003; accepted 29 December 2003; published online 1 April 2004)

The GEKKO/HIPER-laser [N. Miyanaga et al., in Proceedings of the 18th International Conference on Fusion Energy (IAEA, Sorrento, Italy, 2001), IAEA-CN-77] driven shock experiments were characterized in detail for studies on equation-of-state (EOS) at ultrahigh pressures. High-quality shock waves were produced with the bundled 9 laser beams optically smoothed by spectral dispersion technique and Kinoform phase plates. The laser beams were directly focused on targets at up to an intensity of $10^{14} \mathrm{~W} / \mathrm{cm}^{2}$ or higher with a wavelength of $351 \mathrm{~nm}$ and a duration of $2.5 \mathrm{~ns}$. Key issues on dynamic EOS research; the spatial uniformity and temporal steadiness of shock wave were estimated, and the preheating problem was also investigated by measurements of the self-emission and reflectivity from target rear surface. The experimental and analytical methods were validated by using double-step targets consisting of two Hugoniot standard metals. Extreme pressures only accessed in nuclear explosion experiments were generated with reproducibility and good accuracy using the laser direct-drive experimental system, for $\mathrm{Al}, \mathrm{Cu}$, polystyrene, and $\mathrm{Ta}$. It was indicated that new and reliable EOS data at the ultrahigh pressures could be provided for materials ranging from low to high initial density by the laser direct-drive experimental technique. (C) 2004 American Institute of Physics. [DOI: 10.1063/1.1650845]

\section{INTRODUCTION}

Equation-of-state (EOS) data of materials in high pressure regime provide essential information for high-energydensity physics including astrophysics, ${ }^{1}$ geophysics, ${ }^{2}$ and inertial fusion energy (IFE) research. ${ }^{3-5}$ For example, in the IFE researches, compression efficiency and shock structure in fusion capsules critically depend on the EOS., ${ }^{3,4}$ Such hydrodynamic and thermodynamic conditions are evaluated by numerical codes generally employing well-known EOS models. ${ }^{6,7}$ In order to design and address the experiments such as hydrodynamic instabilities ${ }^{8}$ and fast ignition, ${ }^{9}$ accurate EOSs of materials are required.

A sample material is driven to a point on the principal Hugoniot EOS from the initial state (STP state) by a single shock wave. The point represents a final state generated by the shock wave propagating through the sample, which are indicated as a function of pressure, density, and internal energy. With the initial state known, the following conservation relations through the shock front require the measurement of two variables to determine a Hugoniot point,

\footnotetext{
a)Present address: Laboratoire pour l'Utilisation de Lasers Intenses (LULI), Ecole Polytechnique, France; electronic mail: norimasa.ozaki@polytechnique.fr

${ }^{b}$ Also at the Faculty of Engineering, Osaka University, Suita, Osaka 5650871, Japan.
}

$$
\begin{aligned}
& P-P_{0}=\rho_{0} U_{s} u_{p}, \\
& \rho / \rho_{0}=U_{s} /\left(U_{s}-u_{p}\right),
\end{aligned}
$$

and

$$
E-E_{0}=\frac{1}{2}\left(P+P_{0}\right)\left(1 / \rho_{0}-1 / \rho\right),
$$

where $P, \rho$, and $E$ are the pressure, density, and internal energy behind the shock, $U_{s}$ and $u_{p}$ are the shock and particle velocity, respectively, and the zero subscripts denote the initial condition. ${ }^{10}$

Tera-Pascal (TPa) pressures can be achieved only by strong-shock waves driven with high-energy pulse powers such as nuclear explosions. ${ }^{11-13}$ Although high-power laser facilities have demonstrated the potential of investigating the EOS at the ultrahigh pressures, Hugoniot data above $1 \mathrm{TPa}$ are limited to a few cases. ${ }^{14-20}$ Additionally, data for low- $Z$ materials with relatively good accuracy are available in only a few publications. ${ }^{18,19}$

In order to obtain accurate Hugoniot data, high-quality shocks with sufficient spatial uniformity (planarity) and temporal steadiness have to be established. ${ }^{14,21}$ The laser directdrive technique has advantages that enable control of shock pressure profile, high-energy conversion efficiency, and very simple experimental geometry. On the other hand, the preheating effect remains a disadvantage. It is well known that the irradiation by optically smoothed laser beams at a short laser-wavelength is effective to restrain preheating due to 


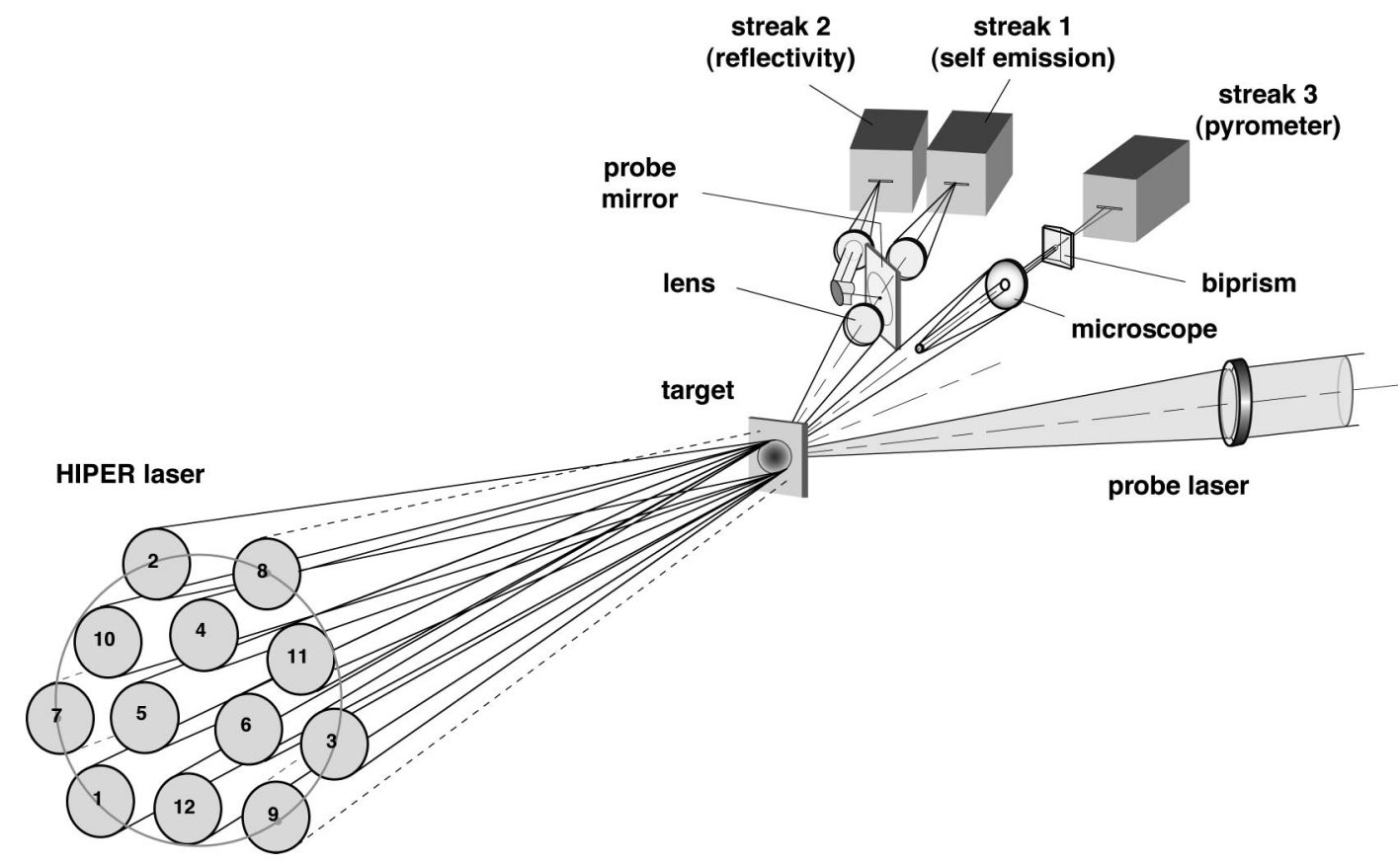

FIG. 1. Experimental schematic. Three streak cameras were used to observe a target rear event at the same time. The numbers 7,8 , and 9 beam are PCL, and the others are SSD beams.

suprathermal electrons. At laser intensities up to a few $10^{14} \mathrm{~W} / \mathrm{cm}^{2}$, preheating is mainly due to thermal x-ray radiation. This type of preheating can be suppressed by use of low- $Z$ ablator and/or thick piston layers. ${ }^{22}$ Once the preheating suppressed which may be verified with a temperature measurement, shock velocity can be measured with good accuracy in laser indirect-drive experiments ${ }^{16}$ and reliable Hugoniot points can be determined by using the impedance matching method (IMM). ${ }^{23}$ Laser-driven absolute EOS measurements require very high-power laser with long pulse duration to generate a main shock loading and a strong backlighter $\mathrm{x}$-ray pulse. ${ }^{19,24}$ Moreover, the alignment relation between the target and the diagnostic (x-ray backlight) is difficult because any tilt of the plane target affects the observed compressibility. ${ }^{25}$ Thus, experiments based on IMM are often preferred.

In this paper, we present experiments to characterize laser-driven EOS studies with the GEKKO/HIPER (High Intensity Plasma Experimental Research) system at the Institute of Laser Engineering (ILE), Osaka University. The spatial and temporal uniformities of shock waves are verified by measuring self-emission and the probe-light reflection from the target rear side. The preheating is evaluated by the rear emission and the reflectivity measurements. The validity of our experiments based on IMM is confirmed with double step targets consisting of aluminum (Al) and copper $(\mathrm{Cu})$ : well-known Hugoniot standard metals. The present experiments show that EOS data at ultrahigh pressures explored previously in nuclear explosion experiments are accessible for any materials with different initial densities.

\section{EXPERIMENT}

A series of experiments was conducted using the HIPER laser facility ${ }^{26}$ which is an irradiation system on the GEKKO
XII (GXII): Nd glass laser system at the ILE. ${ }^{27}$ The HIPER provides one-dimensional compression by smoothed laser beams with short wavelength and high intensity. In the system, 12 beams of the GXII are bundled in an $F / 3$ cone angle. Two beam-smoothing techniques are applied to the system. Three of the 12 beams are partially coherent light (PCL). ${ }^{28}$ The wavelength is $527 \mathrm{~nm}(2 \omega)$ that is the second harmonics of a $1053 \mathrm{~nm}(\omega)$ fundamental. The three beams are used as a foot pulse with low intensity $\left(\sim 10^{12} \mathrm{~W} / \mathrm{cm}^{2}\right)$ to create preplasma in hydrodynamic experiments for IFE research. Other beams are the third harmonic $(3 \omega), 351 \mathrm{~nm}$ wavelength. In these $3 \omega$ beams, smoothing by the spectral dispersion (SSD) technique ${ }^{29}$ is applied. The SSD beams work as a main laser pulse to drive shock waves. Kinoform phase plates (KPPs) ${ }^{30}$ are installed for all beams to obtain a uniform irradiation pattern. In our experiments, only the SSD beams were used to generate a well-defined shock pressure pulse. The temporal behavior of the laser pulse was approximately a square shape in time with a full width at half maximum (FWHM) of $2.5 \mathrm{~ns}$ and a rise and fall time of $100 \mathrm{ps}$. The focal-spot diameter was typically $600 \mu \mathrm{m}$. The laser irradiation spots were routinely monitored by time-resolved and time-integrated $\mathrm{x}$-ray cameras.

A schematic view of the experimental configuration is shown in Fig. 1. Three diagnostic systems were used to measure a target rear-side event at the same time. The first is a measurement of self-emission from shock breakout at the rear surface using a visible streak camera (streak 1 in Fig. 1). The self-emission signals were collected by a $F / 2.8$ lens and were image-relayed on the slit of the streak camera by a microscopic-objective and achromatic lenses. The target rear image was rotated vertically by a dove prism to arrange the steps edge of target on the streak slit. In this optical path, 


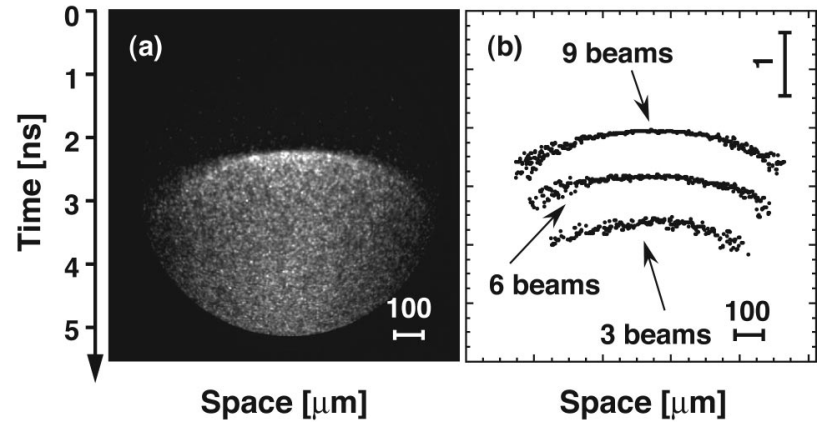

FIG. 2. Streaked images by self-emission measurements with Al planar targets of $40 \mu \mathrm{m}$ thickness. (a) Typical raw data. (b) Dependence of the number of the HIPER beams. Each shock arrival image is intentionally shifted for showing the difference.

band-cut filters were inserted for the $2 \omega$ and $3 \omega$ of the GXII wavelength.

The second diagnostic system was a measurement of the reflection of a probe laser from the target rear side. An injection-seeded Q-switched Nd:YAG (yttrium aluminum garnet) laser was used as the probe light. The maximum energy was $0.7 \mathrm{~J}$ at a wavelength of $532 \mathrm{~nm}$. The original pulse duration was approximately $8 \mathrm{~ns}$ (FWHM) with a Gaussian shape. The probe laser was injected into one end of an optical fiber by a lens and was passed through the fiber to near the vacuum target chamber. Another end of the fiber was coupled with a fiber collimator and a lens, thus the probe light was collimated in front of a focal lens of $F / 3$ in the backlighter beam line of the HIPER system. The YAG probe illuminated the target rear surface using the focal lens of the backlighter beam. Specular reflection of the probe was collected by the same optics utilized in the self-emission measurement. The light was reflected by a laser mirror at the probe wavelength of $532 \mathrm{~nm}$ and was focused on the slit of another streak camera system (streak 2 in Fig. 1). Note that a notch filter (less than $10 \mathrm{~nm}$ bandwidth) for the $532 \mathrm{~nm}$ of YAG was installed to prevent the self-emission measurement from the probe light signal.

The third technique uses a radiation pyrometer based on a color temperature measurement. This system allowed us to obtain spatially, temporally, and spectrally resolved images using one streak camera (streak 3 in Fig. 1). The principle had been presented for the first time by Hall et al. ${ }^{31}$ We could obtain two different spectral images onto the streak slit by using a biprism and two different colored filters. In order to increase sensitivity for color temperature measurements, we choose the spectrum regions of blue (385-469 nm) and ultraviolet $(284-327 \mathrm{~nm})$. The details of this measurement will be described in a future publication.

In our impedance-matching experiments, $\mathrm{Al}$ was used as an EOS standard material. Target structure in irradiation side by the HIPER was classified into two types. First is a plain Al base target for low laser intensity. Second is a two-layered base target with a $\mathrm{CH}$ (polystyrene) ablator to suppress thermal radiation preheating in high laser intensity. The $\mathrm{CH}$ is overcoated with a thin $\mathrm{Al}$ layer of $1000 \AA$ in thickness that prevents direct laser shine-through in the $\mathrm{CH}$. Using a numerical code MY1DL based on a 1D hydrodynamic La- grangian scheme, ${ }^{32}$ we optimized the target thicknesses to maintain steady-shock pressures under our laser conditions. Typically the optimized thicknesses of $\mathrm{Al}$ was between 30 and $70 \mu \mathrm{m}$. To fabricate step targets, an adhesion technique was used with a single molecular membrane coating. ${ }^{33}$ All targets were characterized by a confocal laser $2 \mathrm{D}$-scanning microscope with a minimum scale value in height of $10 \mathrm{~nm}$. Portions of those, in particular EOS measurement targets, were characterized within the area of the HIPER irradiation spot by a 1D-scanning microscope with a height resolution of $1 \mathrm{~nm}$.

\section{SHOCK UNIFORMITY}

\section{A. Spatial uniformity}

In the measurements of Hugoniot EOS, high-quality planar shocks are required. The spatial uniformity was verified in the present experiments with planar $\mathrm{Al}$ targets with 40 to $100 \mu \mathrm{m}$ thicknesses. Figure 2(a) shows the typical streaked image of self-emission from the target rear surface at a laser intensity of $9.2 \times 10^{13} \mathrm{~W} / \mathrm{cm}^{2}$ and a thickness of $40 \mu \mathrm{m}$. Time proceeds from the top to the bottom. The central flat region of shock wave was estimated as over $230 \mu \mathrm{m}$ diameter, with which a variation in breakout time of \pm 8 ps. The flat region was sufficiently wide for EOS experiments with several percent of accuracy. Here, the order of the shock velocity can be considered as several $10 \mathrm{~km} / \mathrm{s}$. The shock wave travels a distance of $100 \mathrm{~nm}$ order in the $8 \mathrm{ps}$. This distance is comparable to the surface roughness of the Al. The variation includes the effect of attenuation due to twodimensional effects, particularly in the left- and right-hand side from the center of the shock wave.

Shock arrival timings in the planar targets are plotted to show the dependence of the number of HIPER beams in Fig. 2(b). Each shock arrival image is intentionally shifted for showing the difference. Here, the shock arrival time was defined as the leading edge mesial point of the shock emission signal. Three beams data show more rugged arrival because the signals are weak; both the pressure and temperature are low. Varying the number of beams does not largely affect the magnitude of the central flat region of driven-shock waves. Consequently, we could obtain repetitive shots because we can divide the number of the HIPER beams to vary the pressure range.

\section{B. Temporal uniformity}

The shock-condition generated on the Hugoniot must be in equilibrium behind the shock wave discontinuity, and must be temporally steady for a duration long enough for the observations. The shape of drive-laser pulse strongly contributes to that of the generated shock pulse. This is advantageous in direct laser-drive technique for easily controlling the pulse shape. Figure 3 shows an example of the pulse shape provided by the laser system in the EOS experimental campaign. The laser pulse was nearly flat-topped with an intensity fluctuation in the saturated zone of less than $\pm 1.4 \%$ over $1.8 \mathrm{~ns}$. 


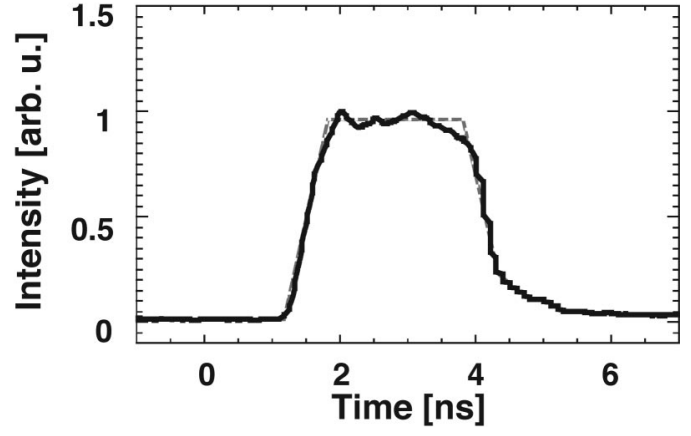

FIG. 3. Temporal pulse shape of the drive laser. The pulse is well defined as a nearly squared shape. Dotted curve is a fit of the squared shape.

The temporal uniformity of the HIPER-driven shock velocity was measured with a wedged target to confirm the steadiness of the shock pressure; the shock breakout time should be proportional to distance along the incline. The wedged targets were made by a precision machining technology. Figure 4 is a streaked image of the rear self-emission using a wedged target (the target structure is also shown). A horizontal dotted double-arrow indicates the central flat region of driven-shock waves mentioned above the subsection. A vertical double-arrow shows the temporal width corresponding to an Al thicknesses between 35 and $68 \mu \mathrm{m}$. The shock-breakout time was delayed linearly in distance along the incline to \pm 18 ps root-mean-square (RMS) in the duration indicated by the vertical arrow, shown as open circles on
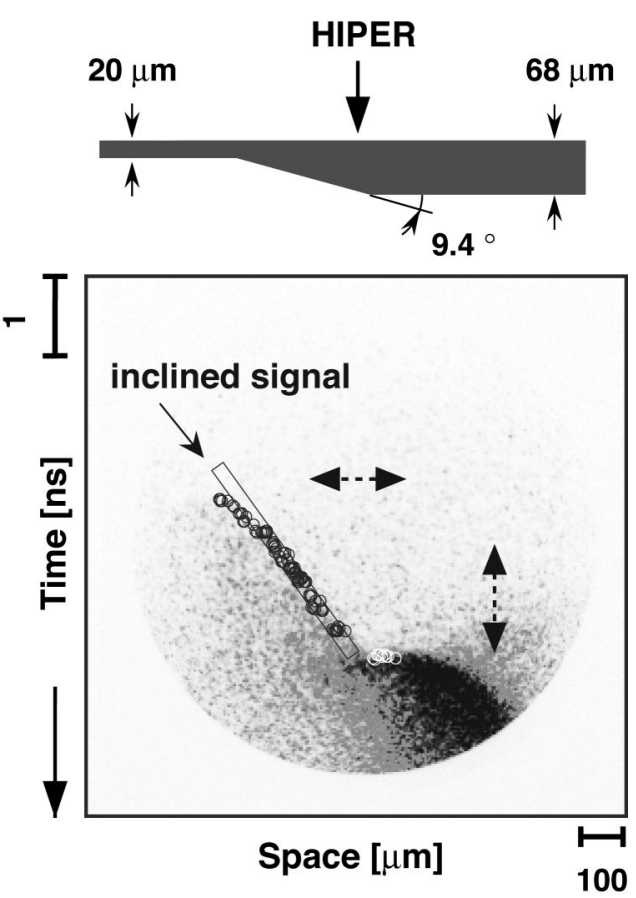

FIG. 4. Typical streaked image by self-emission measurement with a wedged $\mathrm{Al}$ target. The top of the figure is the structure of the wedged target. A horizontal dotted double-arrow indicates the central flat region of drivenshock waves mentioned above the subsection. A vertical double-arrow shows the temporal width corresponding to an $\mathrm{Al}$ thickness between 35 and $68 \mu \mathrm{m}$. Open circles show shock arrival timings that are analyzed in the same way as Fig. 2. Inclined open bar represents a linear curve calculated by least-squares method.

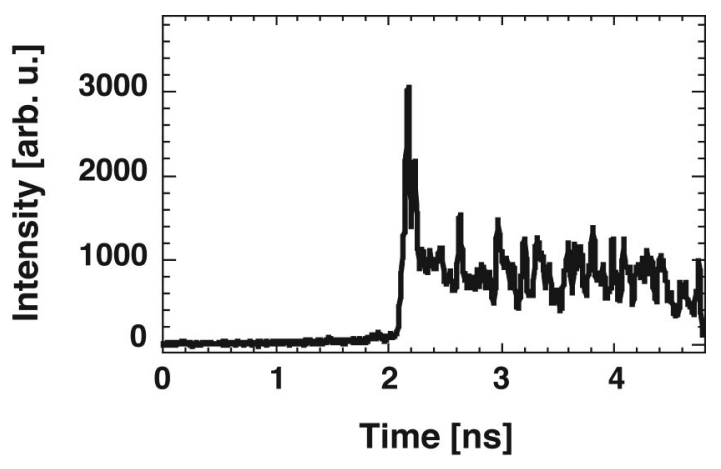

FIG. 5. Typical temporal profile of the rear emission at a $40 \mu \mathrm{m}$ thickness of Al. The drive laser irradiance is $9.2 \times 10^{13} \mathrm{~W} / \mathrm{cm}^{2}$. Significant pre-emission is not detected before the shock breakout.

the image. Shock velocity in this measurement was 28.47 $\pm 0.43 \mathrm{~km} / \mathrm{s}$. The temporal uniformity of the shock wave was hence estimated as $\pm 1.13 \%$. In typical EOS-targets the thickness region of 40-60 $\mu \mathrm{m}$ was used in our experiments for the fair steadiness.

\section{PREHEATING}

When a high-power laser irradiates a material, the laser light is mainly absorbed near the critical density region of the laser-produced plasma. An over-dense plasma has a conduction zone where thermal intense $\mathrm{x}$ rays are emitted. On the other hand, under-dense plasma produce both $\mathrm{x}$ rays and superthermal electrons. These radiations can heat the ambient matters of the cold initial state before the shock front overtakes. The target condition before the shock-front arrival contributes to the final shocked state on the Hugoniot. If the preheating effect significantly changes the initial condition of the sample to a new and unknown state, this effect can consequently obscure or ruin the obtained Hugoniot data. We characterized the preheating temperature level in our experimental conditions. In these experiments, preheating is mainly due to $\mathrm{x}$ rays from the critical density plasma because laser intensity is well below the threshold for any nonlinear parametric instability inducing suprathermal-electron preheating. Moreover, our laser wavelength and uniform irradiation should be effective in reducing the instabilities. ${ }^{22}$

Figure 5 shows the temporal history of the rear emission from a planar Al target represented in Fig. 2(a). This profile is a typical signal from strong-shock breakout, and shows that a well-defined shock is generated. Taking into account the time resolution of the diagnostic system, the measured risetime is shorter than $20 \mathrm{ps}$. After shock passage, the rapid decay denotes that the plasma is cooled due to the expansion into the vacuum without heating by $\mathrm{x}$ rays from the HIPER irradiation side. No significant emission is detected before the shock emergence at the free surface. We therefore believe that the preheating level is less than the detection limit of this measurement, a $0.9 \mathrm{eV}$ blackbody temperature.

The reflected probe-light measurement at the target rear surface is more sensitive to the preheating effect. We define reflectivity as the ratio of reflected light intensity of the shocked target to incident light intensity. An example of re- 


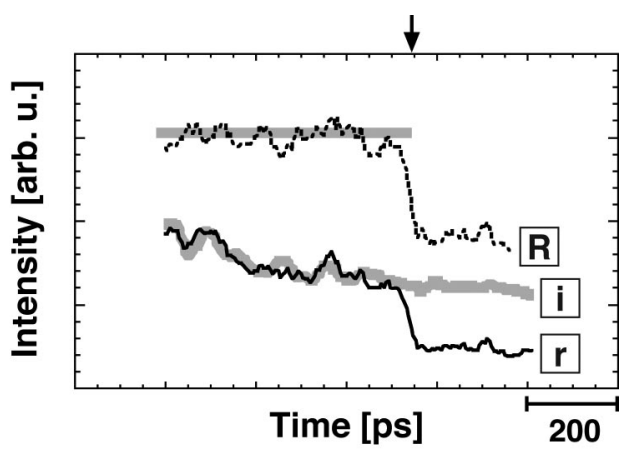

FIG. 6. Typical reflectivity signal (R), and the intensity profile of reflected (r) and incident (i) probe light to determine the reflectivity. Horizontal gray line shows $100 \%$ reflection. Shock arrival time is indicated by an arrow.

flectivity for a $40 \mu \mathrm{m}$ thick $\mathrm{Al}$ is shown as a function of time in Fig. 6. The lower solid thin and gray thick curves indicate the intensity profile of reflected and incident light, respectively, and the upper dotted curve shows the calculated reflectivity. The horizontal bar in the reflectivity curve is a guide to the eye, which denotes $100 \%$ reflection. The drivelaser irradiance was $5.0 \times 10^{13} \mathrm{~W} / \mathrm{cm}^{2}$. Shock breakout time is shown as an arrow. We find that the reflectivity decreases rapidly at shock emergence. No significant decrease of reflectivity was detected before the shock arrival at the rear surface. The decrement before the shock breakout can be estimated as not more than 7\%. This is consistent with about $0.08 \mathrm{eV}$ preheating level. ${ }^{22}$

Moreover, we can present a typical result from velocimetry measurements. Figure 7 is the data obtained with a velocity interferometer system for any reflector (VISAR). ${ }^{34}$ Figure 7(a) is the raw velocimetry image and Fig. 7(b) temporal profile at an arbitrary position. Time proceeds from the left to the right. The experimental conditions are the same as the ones mentioned earlier. The $\mathrm{Al}$ sample thickness was 40 $\mu \mathrm{m}$ and the laser intensity was $6.6 \times 10^{13} \mathrm{~W} / \mathrm{cm}^{2}$. The reflected light discontinuously vanished at the shock arrival timing and no fringe shift was measured before this timing. Here, the fringe constant (sensitivity) of this velocimeter was

(a)

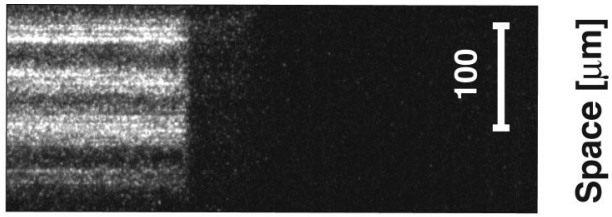

(b)

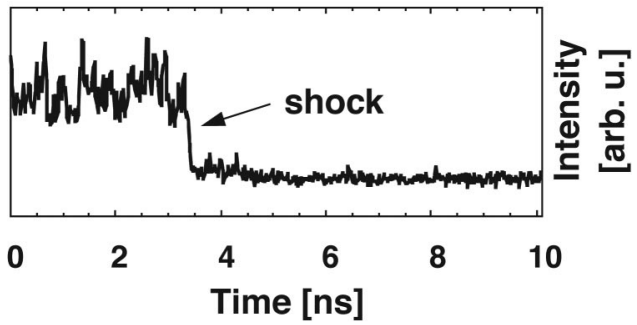

FIG. 7. Typical result with VISAR. (a) Raw streaked image. Time proceeds from the left to the right. (b) The intensity profile is at an arbitrary position. Al base plate was $40 \mu \mathrm{m}$ thickness, and laser intensity was 6.6 $\times 10^{13} \mathrm{~W} / \mathrm{cm}^{2}$.

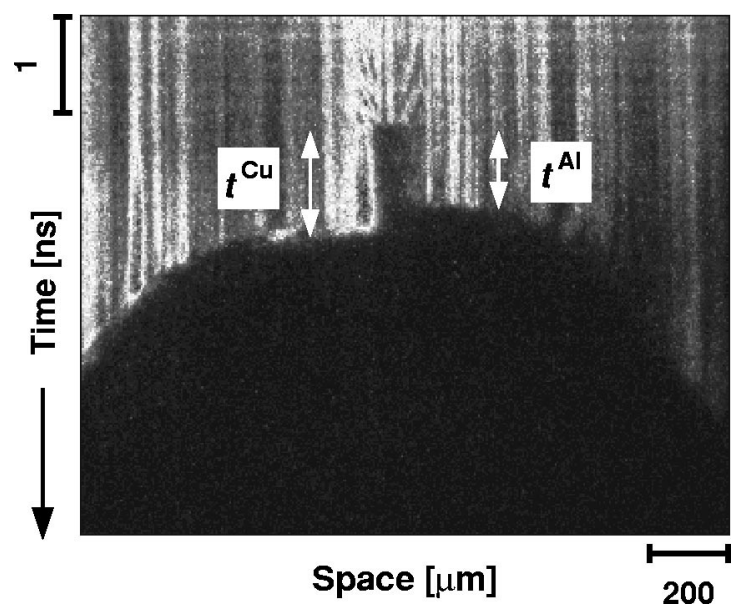

FIG. 8. Streaked image with double standard target by reflected light measurements. The step heights of $\mathrm{Al}$ and $\mathrm{Cu}$ are 19.68 and $19.79 \mu \mathrm{m}$, respectively. The $t^{\mathrm{Al}}$ and $t^{\mathrm{Cu}}$ indicate transit time for the shock traveling through each step, respectively.

$3.164 \mathrm{~km} / \mathrm{s} /$ fringe. The detection limit is better than $1 / 10$ of the fringe constant, $0.316 \mathrm{~km} / \mathrm{s} /$ fringe, which corresponds to an expansion velocity resulting from a temperature of approximately $0.085 \mathrm{eV}$ in $\mathrm{Al}$. This is in agreement with the results of the reflectivity experiments.

Finally, for this temperature level resulting from radiative transfer, the increase of the shock propagating distance due to free surface expansion and of the shock velocity due to temperature increment at a preheated region can be comparable. The competition between these factors can make shock velocity measurements insensitive to the radiation preheating.

\section{VALIDATION}

Our laser-driven IMM experiments were validated with using double-step target consisting of two Hugoniot standards, $\mathrm{Al}$ and $\mathrm{Cu}^{35}$ Figure 8 is an example of typical image obtained by the reflected light measurement. The $\mathrm{Al}$ and $\mathrm{Cu}$ steps are on the right- and left-hand sides, respectively. The time intervals, $t^{\mathrm{Al}}$ and $t^{\mathrm{Cu}}$, correspond to the transit time of the shock propagating through each step, where superscripts denote the material. As the step heights were known, the shock velocity of Al, $U_{s}^{\mathrm{Al}}$, was $26.43 \pm 0.45 \mathrm{~km} / \mathrm{s}$ and that of $\mathrm{Cu}, U_{s}^{\mathrm{Cu}}$, was $19.47 \pm 0.29 \mathrm{~km} / \mathrm{s}$.

The Al Hugoniot has been experimentally and theoretically investigated over a wide range of pressures. Here, we used the SESAME model ${ }^{7}$ of Al to determine the final Hugoniot points. The model is in good agreement with experimental Hugoniot data up to approximately $2 \mathrm{TPa}$. We can also use a simple linear relationship between $U_{s}^{\mathrm{Al}}$ and $u_{p}^{\mathrm{Al}}$ as suggested in Ref. 36. However, the linear relation is not verified in the multi-TPa pressure region, and the accuracy of the two parameters defining this relationship is not high. Additionally, we evaluated that the EOS of electrons should be considered in such pressure region.

The $u_{p}^{\mathrm{Al}}$ and pressure $P^{\mathrm{Al}}$ state corresponding to the measured shock velocity of $26.43 \mathrm{~km} / \mathrm{s}$ were determined as 16.29 $\mathrm{km} / \mathrm{s}$ and $1.16 \mathrm{TPa}$, respectively. When the shock wave 


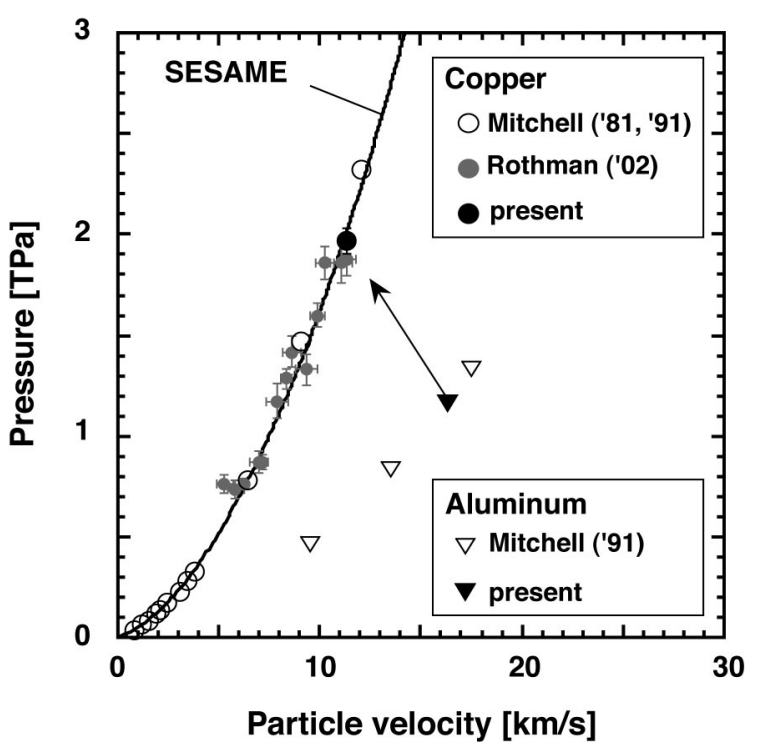

FIG. 9. $\mathrm{Cu}$ and $\mathrm{Al}$ Hugoniot data. Solid circle and triangle are present data. Open circles and triangles are results with nuclear explosions and gas guns (Refs. 13 and 36). Gray circles are $\mathrm{Cu}$ data driven by laser (Ref. 16). Impedance matching process is represented as an arrow.

propagates through the interface between $\mathrm{Al}$ and $\mathrm{Cu}$, a reflected shock travels in the primary shocked Al. The reflected shock condition is given by the intersection of the re-shock curve of $\mathrm{Al}$ and the Rayleigh line of $\mathrm{Cu} ; P=\rho_{0}^{\mathrm{Cu}} U_{s}^{\mathrm{Cu}} u_{p}$ in $P-u_{p}$ plane. The reflected shock curve of Al was calculated based on the SESAME database. Consequently, the particle velocity in $\mathrm{Cu}$ was $11.31 \mathrm{~km} / \mathrm{s}$, and the pressure was $1.97 \mathrm{TPa}$. These values had accuracies better than $3.1 \%$. The determination manner of these errors is described in the Appendix.

The Hugoniot data of $\mathrm{Cu}$ and $\mathrm{Al}$ are plotted in Fig. 9. Circles and triangles denote $\mathrm{Cu}$ and $\mathrm{Al}$ Hugoniot points, respectively. Solid symbol indicates present work. Open circles and triangles are, respectively, the results for $\mathrm{Cu}$ and $\mathrm{Al}$ data from published papers with gas guns $^{36}$ and nuclear explosions. ${ }^{13}$ Three $\mathrm{Cu}$ data points from the highest pressure shown are the results from IMM experiments using nuclear explosions, and others are from absolute experiments using gas guns. The three open circle $\mathrm{Cu}$ data points plotted correspond to the open triangle Al plots, respectively. Gray circles are several examples of $\mathrm{Cu}$ data by Rothman et al. in indirectly driven laser experiments with hohlraum at the AWE, United Kingdom. ${ }^{16}$ The solid curve shows the SESAME Hugoniot EOS for $\mathrm{Cu}$ (SESAME 3332). Our result is fully consistent with this model and previous works using different techniques.

\section{ACCESS TO ULTRAHIGH PRESSURES}

We investigated the laser-energy dependence of drive pressure in Al using step targets (Fig. 10). Open circles denote present results by the emission measurements. It was found that the pressure data are approximately proportional to the two-thirds power of laser energy. This is consistent with a well-known simple scaling law. ${ }^{4}$ The scaling is shown as a gray solid curve in the figure, ${ }^{37}$ indicating that our ex-

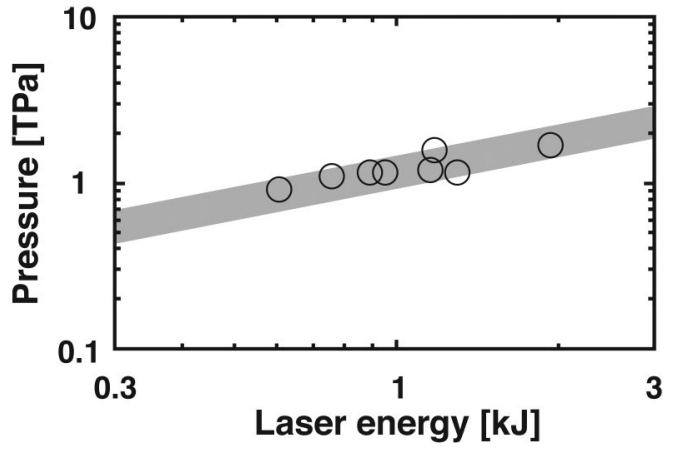

FIG. 10. Laser energy dependence of the generated pressure in Al. The solid gray curve shows a scaling law.

periments can provide systematic results. Here, note that the parameters in the laser irradiation conditions are fixed at a wavelength of $351 \mathrm{~nm}$, a spot diameter of $600 \mu \mathrm{m}$, and a duration of $2.5 \mathrm{~ns}$ (FWHM). Therefore, the dispersion of the data from the scaling primarily results from shot-to-shot ambiguities of these condition parameters and laser energy monitor.

IMM experiments were performed for other materials on the GEKKO/HIPER system. We present results of two materials with very different initial densities. The first is tanta$\operatorname{lum}(\mathrm{Ta})$ which is high- $Z$, with a density of $16.7 \mathrm{~g} / \mathrm{cm}^{3}$. Tantalum is a metal typically used in dynamic high-pressure experiments such as flyers, anvils to reflected-shock for a material, and holders to fill liquid samples. Tantalum is also used as the projectile of multilayered flyer which we developed to generate high pressures with no preheating by laserplasma interactions. ${ }^{32,38}$ The thermodynamic and mechanical properties have been of scientific and applicational interest in the fields of both science and engineering. EOS data of Ta is limited up to about $1 \mathrm{TPa}$ by conventional techniques such as gas guns. This experiment could provide EOS data of the high- $Z$ metal in the multi-TPa pressure region. The reflected shock state of Al was calculated based on the SESAME database, in order to determine the intersection of the reflected shock curve of $\mathrm{Al}$ and the Rayleigh line of Ta. The accuracy of the highest pressure data was better than $4 \%$ in pressure and $5 \%$ in density.

The second is a plastic material which is low- $Z$, $1.04 \mathrm{~g} / \mathrm{cm}^{3}$. Plastics play very important roles as shell materials for inertial-fusion-energy (IFE) fuel capsules and, as described in the first section, have been used extensively in basic IFE experiments. Thus, these EOS data are needed to guide target designs and to analyze experimental results. However, many plastic materials do not have sufficiently good experimental EOS data in the pressure regime of interest. We successfully obtained the Hugoniot data of a low- $Z$ plastic material, polystyrene (PS), up to approximately 2.5 $\mathrm{TPa}$ pressure. Compared with the results of the laser indirectly driven absolute EOS experiments at the Lawrence Livermore National Laboratory (LLNL), ${ }^{19}$ our data, which are laser directly driven relative IMM experiments, are in good agreement with those results. The accuracy of the data was better than $8 \%$ in pressure and $10 \%$ in density. The unloading isentrope of $\mathrm{Al}$ was calculated based on the database, in 


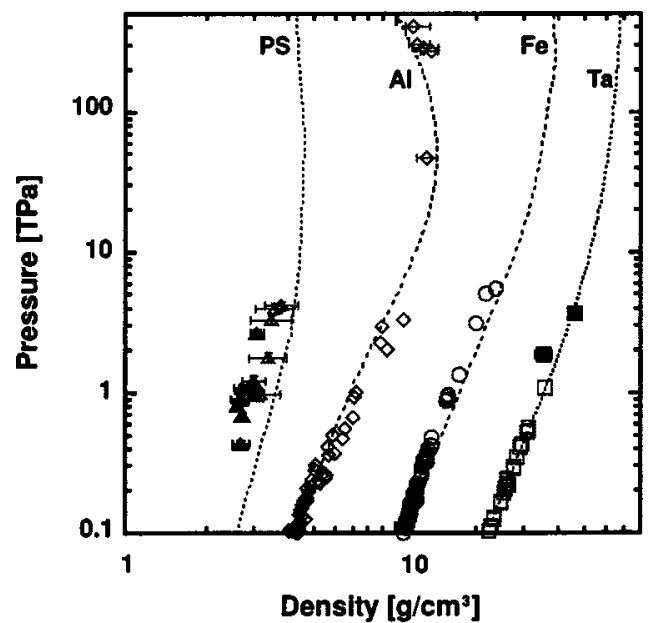

FIG. 11. Summarized Hugoniot data. Solid and open symbols indicate experimental data from present and previous works, respectively. Triangles, diamonds, circles, and squares denote $\mathrm{PS}, \mathrm{Al}, \mathrm{Fe}$, and Ta, respectively (Refs. 12 and 35). Dotted curves are theoretical Hugoniots calculated by the SESAME model (Ref. 7). In PS, open triangles are results from the laser indirectly driven experiments with hohlraum (Ref. 19).

order to determine the intersection of the isentrope curve of $\mathrm{Al}$ and the Rayleigh line of PS. Note that the unloading response of $\mathrm{Al}$ is not as well known as the shock and reshock response so that errors resulting from these measurements may be larger.

Our present experimental results are summarized in Fig. 11, which shows Hugoniot data for several materials. Solid and open symbols indicate experimental data from the present and previously published works, ${ }^{12,35}$ respectively. Triangles, diamonds, circles, and squares denote polystyrene, aluminum, iron $(\mathrm{Fe})$, and tantalum, respectively. Dotted curves show theoretical Hugoniots calculated by the SESAME model. ${ }^{7}$ Only the curve for Ta is an extrapolation from the referenced data because the database does not include Ta. All referenced plots in the TPa region excluding PS were obtained from nuclear explosion experiments. In polystyrene, open triangles are results from the indirectly driven laser experiments using hohlraums. Our data are consistent with the previous data and/or the theoretical model in all cases. The present experiments allowed us to obtain new EOS data for materials with both low and high initial densities, $1.04 \mathrm{~g} / \mathrm{cm}^{3}$ in PS and $16.7 \mathrm{~g} / \mathrm{cm}^{3}$ in Ta. This demonstrates that the GEKKO/HIPER-driven EOS experimental system can provide ultrahigh pressure data, previously explored only in nuclear explosion experiments for several materials with different initial densities.

\section{CONCLUSIONS AND SUMMARY}

In conclusion, we have presented experiments to characterize GEKKO/HIPER-driven shock waves for studies of EOS at ultrahigh pressures. In order to generate high-quality shock waves in the laser direct-irradiation scheme, we used optically smoothed laser beams by a spectral dispersion technique and Kinoform phase plates. The laser intensity was up to $10^{14} \mathrm{~W} / \mathrm{cm}^{2}$ or higher with a wavelength of $351 \mathrm{~nm}$ and a squared pulse of $2.5 \mathrm{~ns}$ duration.
The key issues for laser-driven EOS experiments were confirmed with planar and wedged Al targets and with selfemission and reflection measurements. Shock planarity was over $230 \mu \mathrm{m}$ in the central flat region, which is sufficient for the spatial scale of our EOS experiments. The shock pressure was typically $\pm 1.3 \%$ steady for approximately 2 ns corresponding to the shock propagating time between target thicknesses of 40 and $60 \mu \mathrm{m}$. Preheating effects were investigated from the temporal profile of the emission and reflectivity; significant evidence for preheating was not detected before shock arrivals at the target rear surface. The temperature level was predicted as not more than $0.08 \mathrm{eV}$ for intensities to approximately $7 \times 10^{13} \mathrm{~W} / \mathrm{cm}^{2}$. This suggested that radiation preheating did not strongly affect the shock velocity measurements. Note that the characterization of the effects at a wider range of laser intensities, such as an intensity of more than $10^{14} \mathrm{~W} / \mathrm{cm}^{2}$, is surely worthy. We should continue to perform experimental investigations and compare results with numerical simulations including different opacity models. This will allow us to enhance the accuracy of the higher pressure data and to apply the system to new materials and fields.

We verified our experimental technique based on the impedance matching method using double-step target consisting of Hugoniot standard metals of $\mathrm{Al}$ and $\mathrm{Cu}$. The result was in agreement with previous experimental data by different tools and an EOS model. This assures the validity of EOS experiments for unknown materials.

Extreme pressures in Al standard were created with systematization and reproducibility. Additionally, new Hugoniot points up to $3 \mathrm{TPa}$ were obtained for materials with very different initial densities using the experimental system. The data support past works and/or theoretical models. These results indicate that the laser directly driven experiments can provide multi-TPa data for arbitrary materials previously accessible only in nuclear explosion experiments.

\section{ACKNOWLEDGMENTS}

The authors gratefully acknowledge the valuable support for the experiments by the GXII technical crews and scientists at the ILE. In particular, the authors would like to thank H. Shiraga and K. Nishihara for fruitful discussions, K. Suzuki, S. Urushihara, H. Asahara, and N. Morio for operating the GXII, O. Maegawa, K. Shimada, T. Kuwamoto, and Y. Hori for diagnostic supports, and Y. Kimura, K. Nagai, T. Norimatsu and their group for target fabrications. The authors would like to thank K. Okada (AIST) for useful comments and help. Two authors (T.O. and N.O.) would like to thank M. Nakano and T. Kataoka (Osaka University) for fruitful discussions and useful comments, and Y. Igarashi (MSL), S. Fujioka, and T. Shiota for valuable experimental and analytical support, and Y. Tohyama and $\mathrm{H}$. Nishimura (ILE) for preparing diagnostic instruments. One of the authors (N.O.) would like to thank M. Koenig (Ecole Polytechnique) for providing EOS data.

This work was performed under the auspices of the Japan Science and Technology Corporation (JST) by Osaka University under Contract No. A2-12308020. 


\section{APPENDIX: ERROR ESTIMATION}

In this appendix, we describe error analysis of the EOS data in this paper.

\section{Shock velocity error}

The shock velocity is an experimentally measured variable. Uncertainties are contributed by the variation of step height, $\Delta h$, and shock transit time, $\Delta t$, in a target step. Taking into account those values depending on the accuracy of calibration in metrology and diagnostics, the velocity error can be expressed as the root sum square of those errors;

$$
\frac{\Delta U_{s}}{U_{s}}=\left\{\left(\frac{\Delta h}{h}\right)^{2}+\left(\frac{\Delta c_{\mathrm{mscp}}}{c_{\mathrm{mscp}}}\right)^{2}+\left(\frac{\Delta t}{t}\right)^{2}+\left(\frac{\Delta c_{\text {strk }}}{c_{\text {strk }}}\right)^{2}\right\}^{1 / 2},
$$

where $\left(\Delta c_{\text {mscp }} / c_{\text {mscp }}\right)$ and $\left(\Delta c_{\text {strk }} / c_{\text {strk }}\right)$ indicate the calibration accuracy of microscope and streak camera. The error of step height is determined by the variation of height due to the target surface configuration and roughness. The error of shock transit time results from the variation of the shock arrival signal due to the target surface roughness, the shock planarity, and the pressure distribution in the shock front. Furthermore, strictly speaking, the shock steadiness error must be added to the error of $U_{s}$, because $U_{s}$ is an averaged velocity for the transit time. Finally, the error of $U_{s}$ can be defined as

$$
\begin{aligned}
\frac{\Delta U_{s}}{U_{s}}= & \left\{\left(\frac{\Delta h}{h}\right)^{2}+\left(\frac{\Delta c_{\mathrm{mscp}}}{c_{\mathrm{mscp}}}\right)^{2}+\left(\frac{\Delta t}{t}\right)^{2}+\left(\frac{\Delta c_{\text {strk }}}{c_{\text {strk }}}\right)^{2}\right. \\
& \left.+\left(\frac{\Delta U_{\mathrm{tmp}}}{U_{\mathrm{tmp}}}\right)^{2}\right\}^{1 / 2},
\end{aligned}
$$

where $U_{\mathrm{tmp}}$ is the temporal constancy of the shock.

The error of microscope calibration was negligible because of the high accuracy compared with that of step height variation, and that of streak camera was less than $0.22 \%$. The error of shock constancy was estimated as better than $1.2 \%$ from the results of the wedged target. We can add the preheating effect to the error in shock velocity. However, as described in Sec. IV, we evaluated that the influence is not strong for the velocity measurements. For instance, in the case of a $\mathrm{Cu}$ shot, we determined the error of the velocity as

$$
\begin{aligned}
\frac{\Delta U_{s}^{\mathrm{Cu}}}{U_{s}^{\mathrm{Cu}}}= & \left\{(0.14 \%)^{2}+(0 \%)^{2}+(0.93 \%)^{2}+(0.16 \%)^{2}\right. \\
& \left.+(1.13 \%)^{2}\right\}^{1 / 2}=1.46 \%
\end{aligned}
$$

\section{Particle velocity}

The unknown particle velocity, $u_{p}^{\mathrm{unk}}$, is determined as the intersection of the re-shock curve (or the unloading isentrope) of $\mathrm{Al}$ and the Rayleigh line of unknown sample. The re-shock and isentropic curve in $\mathrm{Al}$ are uniquely calculated from the measured shock velocity of $\mathrm{Al}$ by using the SESAME model. Therefore, the uncertainties of those curves are contributed to by the variation of the shock velocity, $\Delta U_{s}^{\mathrm{Al}}$, and numerical that of the EOS model, $\Delta S_{\mathrm{ESAME}}$. The uncertainty of the Rayleigh line is contributed to by the variation of $U_{s}^{\mathrm{unk}}$ and that of the initial density of the unknown sample, $\rho_{0}^{\text {unk }}$. First, the error of $u_{p}^{\text {unk }}$ is given by

$$
\begin{aligned}
\frac{\Delta u_{p}^{\mathrm{unk}}}{u_{p}^{\mathrm{unk}}}= & \left\{\left(\frac{\Delta \rho_{0}^{\mathrm{unk}}}{\rho_{0}^{\mathrm{unk}}}\right)^{2}+\left(\frac{\Delta U_{s}^{\mathrm{unk}}}{U_{s}^{\mathrm{unk}}}\right)^{2}+\left(\frac{\Delta U_{s}^{\mathrm{Al}}}{U_{s}^{\mathrm{AI}}}\right)^{2}\right. \\
& \left.+\left(\frac{\Delta S_{\text {ESAME }}}{S_{\text {ESAME }}}\right)^{2}\right\}^{1 / 2} .
\end{aligned}
$$

Here, the initial density error should include the variation of the density due to preheating effect. Therefore, we rewrite this equation to

$$
\begin{aligned}
\frac{\Delta u_{p}^{\mathrm{unk}}}{u_{p}^{\mathrm{unk}}}= & \left\{\left(\frac{\Delta \rho_{0}^{\mathrm{unk}}}{\rho_{0}^{\mathrm{unk}}}\right)^{2}+\left(\frac{\Delta \rho_{\mathrm{pht}}^{\mathrm{unk}}}{\rho_{\mathrm{pht}}}\right)^{2}+\left(\frac{\Delta U_{s}^{\mathrm{unk}}}{U_{s}^{\mathrm{unk}}}\right)^{2}+\left(\frac{\Delta U_{s}^{\mathrm{Al}}}{U_{s}^{\mathrm{Al}}}\right)^{2}\right. \\
& \left.+\left(\frac{\Delta S_{\mathrm{ESAME}}}{S_{\mathrm{ESAME}}}\right)^{2}+\left(\frac{\Delta \rho_{0}^{\mathrm{Al}}}{\rho_{0}^{\mathrm{Al}}}\right)^{2}+\left(\frac{\Delta \rho_{\mathrm{pht}}^{\mathrm{Al}}}{\rho_{\mathrm{pht}}^{\mathrm{Al}}}\right)^{2}\right\}^{1 / 2} .
\end{aligned}
$$

The initial density errors were less than $0.1 \%$. Assuming that $\mathrm{Al}$ free surface expands by $0.3 \mathrm{~km} / \mathrm{s}$ at the detection limit level of preheating temperature, we can expect that the decreases of density in the Al base plate is approximately less than $0.9 \%$ and that in the step is much less than it through the experimental campaign. As the preheating level of unknowns was not measured sufficiently, this value was applied to the unknown density error. This amount of preheating temperature is probably an overestimate for unknown materials and $\mathrm{Al}$. No errors from the standard $\mathrm{Al}$ EOS in the SESAME database are used, because we have no reasonable numbers for this. As the SESAME table is based on empirical data, it can contain uncertainties in the off-Hugoniot response, especially the unloading isentrope, at high pressures. This may significantly increase the errors in the EOS of sample materials.

\section{Pressure and density}

As the final Hugoniot pressure and density of unknown are calculated from Eqs. (1) and (2), respectively, at first each error is expressed as follows:

$$
\frac{\Delta P^{\mathrm{unk}}}{P^{\mathrm{unk}}}=\left\{\left(\frac{\Delta \rho_{0}^{\mathrm{unk}}}{\rho_{0}^{\mathrm{unk}}}\right)^{2}+\left(\frac{\Delta U_{s}^{\mathrm{unk}}}{U_{s}^{\mathrm{unk}}}\right)^{2}+\left(\frac{\Delta u_{p}^{\mathrm{unk}}}{u_{p}^{\mathrm{unk}}}\right)^{2}\right\}^{1 / 2}
$$

and

$$
\begin{aligned}
\frac{\Delta \rho^{\mathrm{unk}}}{\rho^{\mathrm{unk}}}= & {\left[\left(\frac{\Delta \rho_{0}^{\mathrm{unk}}}{\rho_{0}^{\mathrm{unk}}}\right)^{2}+\left(\frac{u_{p}^{\mathrm{unk}}}{U_{s}^{\mathrm{unk}}-u_{p}^{\mathrm{unk}}}\right)^{2}\left\{\left(\frac{\Delta U_{s}^{\mathrm{unk}}}{U_{s}^{\mathrm{unk}}}\right)^{2}\right.\right.} \\
& \left.\left.+\left(\frac{\Delta u_{p}^{\mathrm{unk}}}{u_{p}^{\mathrm{unk}}}\right)^{2}\right\}\right]^{1 / 2} .
\end{aligned}
$$

The initial density error should include the effect due to preheating. Finally, these equations can be rewritten as

$$
\begin{aligned}
\frac{\Delta P^{\mathrm{unk}}}{P^{\mathrm{unk}}}= & \left\{\left(\frac{\Delta \rho_{0}^{\mathrm{unk}}}{\rho_{0}^{\mathrm{unk}}}\right)^{2}+\left(\frac{\Delta \rho_{\mathrm{pht}}^{\mathrm{unk}}}{\rho_{\mathrm{pht}}^{\mathrm{unk}}}\right)^{2}+\left(\frac{\Delta U_{s}^{\mathrm{unk}}}{U_{s}^{\mathrm{unk}}}\right)^{2}\right. \\
& \left.+\left(\frac{\Delta u_{p}^{\mathrm{unk}}}{u_{p}^{\mathrm{unk}}}\right)^{2}\right\}^{1 / 2}
\end{aligned}
$$


and

$$
\begin{aligned}
\frac{\Delta \rho^{\mathrm{unk}}}{\rho^{\mathrm{unk}}}= & {\left[\left(\frac{\Delta \rho_{0}^{\mathrm{unk}}}{\rho_{0}^{\mathrm{unk}}}\right)^{2}+\left(\frac{\Delta \rho_{\mathrm{pht}}^{\mathrm{unk}}}{\rho_{\mathrm{pht}}^{\mathrm{unk}}}\right)^{2}\right.} \\
& \left.+\left(\frac{u_{p}^{\mathrm{unk}}}{U_{s}^{\mathrm{unk}}-u_{p}^{\mathrm{unk}}}\right)^{2}\left\{\left(\frac{\Delta U_{s}^{\mathrm{unk}}}{U_{s}^{\mathrm{unk}}}\right)^{2}+\left(\frac{\Delta u_{p}^{\mathrm{unk}}}{u_{p}^{\mathrm{unk}}}\right)^{2}\right\}\right]^{1 / 2} .
\end{aligned}
$$

${ }^{1}$ W. J. Nellis, M. Ross, and N. C. Holmes, Science 269, 1249 (1995); D. Saumon, G. Chabrier, and H. M. Van Horn, Astrophys. J., Suppl. Ser. 99, 713 (1995); W. J. Nellis, Planet. Space Sci. 48, 671 (2000).

${ }^{2}$ R. G. McQueen and S. P. Marsh, J. Geophys. Res. 71, 1751 (1966); C. S. Yoo, N. C. Holmes, M. Ross, D. J. Webb, and C. Pike, Phys. Rev. Lett. 70, 3931 (1993); S. K. Saxena, G. Shen, and P. Lazor, Science 264, 405 (1994); V. N. Zharkov, Phys. Earth Planet. Inter. 109, 1 (1998).

${ }^{3}$ S. W. Haan, S. M. Pollaine, J. D. Lindl et al., Phys. Plasmas 2, 2480 (1995).

${ }^{4}$ J. D. Lindl, Phys. Plasmas 2, 3933 (1995).

${ }^{5}$ T. R. Dittrich, S. W. Hann, M. M. Marinak et al., Phys. Plasmas 6, 2164 (1999).

${ }^{6}$ R. M. More, K. H. Warren, D. A. Young, and G. B. Zimmerman, Phys. Fluids 31, 3059 (1988).

${ }^{7}$ SESAME, the LANL equation of state database, Los Alamos National Laboratory, LA-UR-92-3407 (1992). Copies may be ordered from the National Technical Information Service, Springfield, Virginia 22161.

${ }^{8}$ R. J. Taylor, J. P. Dahlburg, A. Iwase, J. H. Gardner, D. E. Fyfe, and O. Willi, Phys. Rev. Lett. 76, 1643 (1996); H. Azechi, M. Nakai, K. Shigemori, N. Miyanaga, H. Shiraga, H. Nishimura, M. Honda, R. Ishizaki, J. G. Wouchuk, H. Takabe, K. Nishihara, and K. Mima, Phys. Plasmas 4, 4079 (1997); E. Wolfrum, J. Wark, J. Zhang et al., ibid. 5, 227 (1998).

${ }^{9}$ K. A. Tanaka, R. Kodama, K. Mima et al., Phys. Plasmas 10, 1925 (2003).

${ }^{10}$ Y. B. Zel'dovich and Y. P. Raizer, Physics of Shock Waves and HighTemperature Hydrodynamic Phenomena (Academic, New York, 1966).

${ }^{11}$ C. E. Ragan III, Phys. Rev. A 25, 3360 (1982); W. J. Nellis, J. A. Moriarty, A. C. Mitchell, and N. C. Holmes, J. Appl. Phys. 82, 2225 (1997); W. J. Nellis, J. A. Moriarty, A. C. Mitchell, M. Ross, R. G. Dandrea, N. W. Ashcroft, N. C. Holmes, and G. R. Gathers, Phys. Rev. Lett. 60, 1414 (1988).

${ }^{12}$ A. Vladimirov, JETP Lett. 39, 82 (1984).

${ }^{13}$ A. C. Mithcell, W. J. Nellis, J. A. Moriarty, R. A. Heinle, N. C. Holmes, R. E. Tipton, and G. W. Repp, J. Appl. Phys. 69, 2981 (1991).

${ }^{14}$ M. Koenig, B. Faral, J. M. Boudenne, D. Batani, A. Benuzzi, and S. Bossi, Phys. Rev. E 50, R3314 (1994).

${ }^{15}$ M. Koenig, B. Faral, J. M. Boudenne, D. Batani, A. Benuzzi, S. Bossi, C. Remond, J. P. Perrine, M. Temporal, and S. Atzeni, Phys. Rev. Lett. 74, 2260 (1995)

${ }^{16}$ A. M. Evans, N. J. Freeman, P. Graham, C. J. Horsfield, S. D. Rothman, B. R. Thomas, and A. J. Tyrrell, Laser Part. Beams 14, 113 (1996); S. D. Rothman, A. M. Evans, C. J. Horsfield, P. Graham, and B. R. Thomas, Phys. Plasmas 9, 1721 (2002).

${ }^{17}$ A. Benuzzi, Th. Löwer, M. Koenig, B. Faral, D. Batani, D. Beretta, C. Danson, and D. Pepler, Phys. Rev. E 54, 2162 (1996).

${ }^{18}$ M. Koenig, A. Benuzzi, B. Faral, J. Krishnan, J. M. Boudenne, T. Jalinaud, C. Remond, A. Decoster, D. Batani, D. Beretta, and T. A. Hall, Appl. Phys. Lett. 72, 1033 (1998).

${ }^{19}$ R. Cauble, T. S. Perry, D. R. Bach, K. S. Budil, B. A. Hammel, G. W.
Collins, D. M. Gold, J. Dunn, P. Celliers, L. B. Da Silva, M. E. Foord, R. J. Wallace, R. E. Stewart, and N. C. Woolsey, Phys. Rev. Lett. 80, 1248 (1998)

${ }^{20}$ D. Batani, A. Morelli, M. Tomasini et al., Phys. Rev. Lett. 88, 235502 (2002).

${ }^{21}$ Th. Löwer, R. Sigel, K. Eidmann, I. B. Földes, S. Hüller, J. Massen, G. D. Tsakiris, S. Witkowski, W. Preuss, H. Nishimura, H. Shiraga, Y. Kato, S. Nakai, and T. Endo, Phys. Rev. Lett. 72, 3186 (1994).

${ }^{22}$ A. Benuzzi, M. Koenig, B. Faral, J. Krishnan, F. Pisani, D. Batani, S. Bossi, D. Beretta, T. Hall, S. Ellwi, S. Höller, J. Honrubia, and N. Grandjouan, Phys. Plasmas 5, 1 (1998).

${ }^{23}$ High-Velocity Impact Phenomena, edited by R. Kinslow (Academic, New York, 1970).

${ }^{24}$ L. B. Da Silva, P. Celliers, G. W. Collins, K. S. Budil, N. C. Holmes, T. W. Barbee, Jr., B. A. Hammel, J. D. Kilkenny, R. J. Wallace, M. Ross, R. Cauble, A. Ng, and G. Chiu, Phys. Rev. Lett. 78, 483 (1997).

${ }^{25}$ W. J. Nellis, Phys. Rev. Lett. 89, 165502 (2002).

${ }^{26}$ N. Miyanaga, M. Nakatsuka, H. Azechi, H. Shiraga, T. Kanabe, H. Asahara, H. Daido, H. Fujita, and K. Fujita, Proceedings of the 18th International Conference on Fusion Energy (IAEA, Sorrento, Italy, 2001), IAEACN-77.

${ }^{27}$ C. Yamanaka, in Proceedings of the Inertial Fusion Sciences and Applications 99, Bordeaux, 1999, edited by C. Labaune, W. J. Hogan, and K. A. Tanaka (Elsevier, Paris, 2000), p. 19.

${ }^{28}$ H. Nakano, K. Tsubakimoto, N. Miyanaga, M. Nakatsuka, T. Kanabe, H. Azechi, T. Jitsuno, and S. Nakai, J. Appl. Phys. 73, 2122 (1993).

${ }^{29}$ S. Skupsky, R. W. Short, T. Kessler, R. S. Craxton, S. Letzring, and J. M. Soures, J. Appl. Phys. 66, 3456 (1989).

${ }^{30}$ R. M. Stevenson, M. J. Norman, T. H. Bett, D. A. Pepler, C. N. Danson, and I. N. Ross, Opt. Lett. 19, 363 (1994).

${ }^{31}$ T. A. Hall, A. Benuzzi, D. Batani, D. Beretta, S. Bossi, B. Faral, M. Koenig, J. Krishnan, Th. Löwer, and M. Mahdieh, Phys. Rev. E 55, R6356 (1997).

${ }^{32}$ T. Kadono, M. Yoshida, E. Takahashi, I. Matsushima, Y. Owadano, N. Ozaki, K. Fujita, M. Nakano, K. A. Tanaka, H. Takenaka, and K. Kondo, J. Appl. Phys. 88, 2943 (2000).

${ }^{33}$ K. Nagai, T. Norimatsu, T. Yamanaka, T. Nishibe, N. Ozaki, K. Takamatsu, T. Ono, M. Nakano, and K. A. Tanaka, Jpn. J. Appl. Phys., Part 2 41, L1184 (2002); K. Takamatsu, N. Ozaki, K. A. Tanaka et al., Phys. Rev. E 67, 056406 (2003).

${ }^{34}$ L. M. Barker and R. E. Hollenbach, J. Appl. Phys. 43, 4669 (1972).

${ }^{35}$ A. V. Bushman, I. V. Lomonosov, and V. E. Fortov, Equations of State for Metals at High Energy Density (Inst. Chem. Phys., Chernogolovka, 1992) (in Russian).

${ }^{36}$ A. C. Mitchell and W. J. Nellis, J. Appl. Phys. 52, 3363 (1981).

${ }^{37}$ The ablation pressure $P_{a b l}$ in TPa was approximately given by the scaling: $P_{a b l} \approx 0.86\left(I / 10^{14}\right)^{2 / 3} \lambda^{-2 / 3}(A / 2 Z)^{1 / 3}$, where $I$ is a deposited laser intensity in $\mathrm{W} / \mathrm{cm}^{2}, \lambda$ is the laser wavelength in $\mu \mathrm{m}$, and $A$ and $Z$ are the mass and atomic number of target material, respectively. In Fig. 10, about $\pm 8 \%$ tolerance is given to the scaling. The tolerance corresponds to $10 \%$ error of laser parameters consisting of laser energy, spot size and pulse duration, being approximately agreement with previous published experimental data; S. Atzeni, Plasma Phys. Controlled Fusion 29, 1535 (1987); J. D. Kilkenny, Laser Program Annual Progress Report (Lawrence Livermore National Laboratory, Livermore CA, 1987), Report No. UCRL 50021-86, p. 3.

${ }^{38}$ K. A. Tanaka, M. Hara, N. Ozaki, Y. Sasatani, S. I. Anisimov, K. Kondo, M. Nakano, K. Nishihara, H. Takenaka, M. Yoshida, and K. Mima, Phys. Plasmas 7, 676 (2000); N. Ozaki, Y. Sasatani, K. Kishida et al., J. Appl. Phys. 89, 2571 (2001). 